\title{
Video intervention for the psychiatric waiting room: proof-of-concept randomised controlled trial of RESOLVE (Relaxation Exercise, SOLVing problem and cognitive Errors)
}

Farooq Naeem, Tariq Munshi, David Gratzer, David Rodie, Muhammad Irfan, Sanjay Rao, Nusrat Husain, Saeed Farooq, Marcos Sanches, Muhammad Ayub and Tania Lecomte

\section{Background}

The waiting room in psychiatric services can provide an ideal setting for offering evidence-based psychological interventions that can be delivered through electronic media. Currently, there is no intervention available that have been developed or tested in mental health.

\section{Aims}

This proof-of-concept study aimed to evaluate a pilot design of RESOLVE (Relaxation Exercise, SOLving problem and cognitive Errors) to test the procedure and obtain outcome data to inform future, definitive trials (trial registration at Clinicaltrials.gov NCT02536924, REB Number: PSIY-477-15).

\section{Method}

Forty participants were enrolled and equally randomised to the intervention, RESOLVE plus treatment as usual arm (TAU), or to a control group (TAU only). Those in the intervention group watched RESOLVE in a room adjacent to the waiting area. Participants in the control received routine care. Outcome measures included the Hospital Anxiety and Depression Scale; the Clinical Outcomes in Routine Evaluations outcome measure; and the World Health Organization Disability Assessment schedule. These measures were performed by a masked assessor at baseline and at 6-week follow-up. Additionally, we measured the number of contacts with mental health services during the prior 4 weeks. Both intention-to-treat and per protocol analyses were performed.

\section{Results}

The study proved feasible. We were able to recruit the required number of participants. There was a statistically significant improvement in depression $(P<0.001)$, anxiety $(P<0.001)$, general psychopathology $(P<0.001)$ and disability $(P=0.0361)$ in favour of the intervention group. People in the intervention group were less likely to contact the service $(P=0.012)$ post-intervention.

\section{Conclusions}

Findings provide preliminary evidence that evidence-based psychosocial interventions can be delivered through electronic media in a waiting-room setting. The outcome data from this study will be used for future definitive trials.

\section{Declaration of interest}

None.

\section{Keywords}

Waiting room; problem solving; video intervention; breathing; cognitive errrors.

\section{Copyright and usage}

(C) The Royal College of Psychiatrists 2019. This is an Open Access article, distributed under the terms of the Creative Commons Attribution-NonCommercial-NoDerivatives licence (http://creativecommons.org/licenses/by-nc-nd/4.0/), which permits noncommercial re-use, distribution, and reproduction in any medium, provided the original work is unaltered and is properly cited. The written permission of Cambridge University Press must be obtained for commercial re-use or in order to create a derivative work.

\section{Background}

The healthcare waiting-room setting presents an opportunity to offer a variety of interventions to patients. The average amount of time people spend in a physician's waiting room varies between 19 and 50 min. ${ }^{1,2}$ Sherwin et al suggest that waiting rooms can be used for a variety of interventions, for example to complete validated questionnaires, question prompt sheets or brief training, and provide patient education material and decision aids. ${ }^{3}$

Only limited literature is available on the use and evaluation of specific interventions developed for waiting rooms. Few studies have assessed the impact of interventions provided in the waiting room. These studies are mostly limited to health education delivered in a variety of formats, ranging from pamphlets to audiovisual aids and in a variety of healthcare settings. ${ }^{4-10}$

Evidence suggests that single-session interventions can be effective in managing a variety of mental and emotional health problems. ${ }^{11-17}$ There is also evidence that a brief video intervention might help with mental health problems. ${ }^{18}$ Therefore, we believe that waiting-room interventions for psychiatric settings can go beyond simple educational material and psychiatric interventions can be offered through digital media, including audiovisual material.

We performed a literature search to identify studies of waitingroom interventions in mental health. We did not find a published report on the development or evaluation of a waiting-room intervention explicitly developed for psychiatric patients. We developed a waiting room video intervention, RESOLVE (Relaxation Exercise, SOLving problem and cognitiVe Errors), for the psychiatric waiting room. This paper reports the results of a proof-of-concept study that evaluated the intervention in a crisis clinic.

\section{Development of RESOLVE}

RESOLVE was developed following clinical observations by us, informal discussions with clinical colleagues and experts in the field. Clinical observations suggest that employing relaxation strategies, analysing the problem and better understanding our thought processes are useful first steps towards feeling better. We decided to focus on problem-solving therapy (PST) as this approach can be taught using electronic media. A video was produced based on 


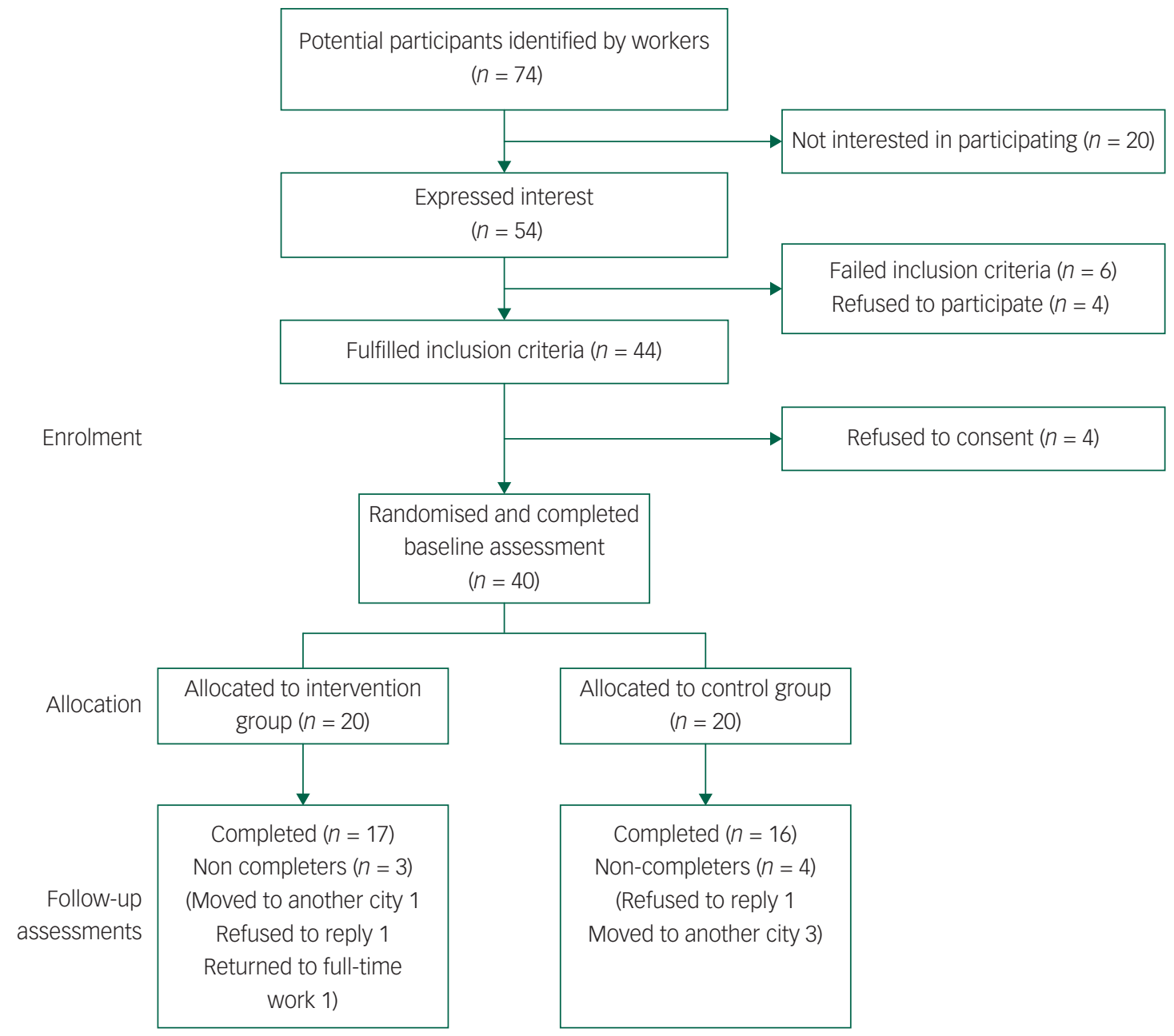

Fig. 1 Consort flow diagram of the trial.

this problem-solving approach. PST is effective for a variety of mental health problems. ${ }^{19,20}$ People in distress often have difficulties thinking clearly about their situation and what might help to improve it and a problem-solving approach may be useful in helping people presenting to crisis or emergency services. ${ }^{21}$ One reason for people to seek help from psychiatric crisis services is that their ability to cope and solve problems on their own has been insufficient. ${ }^{22}$ Mindfulness-based breathing exercises have also been found to be useful in helping those with mental health problems. ${ }^{23}$ There is sufficient evidence to suggest a role for breathing exercises in dealing with stress, anxiety and negative affect. $^{24}$ According to cognitive theory, our dysfunctional thoughts lead to extreme emotions. ${ }^{25} \mathrm{Fu}$ et al $(2013)^{16}$ reported that a single session of cognitive bias modification training could reduce negative interpretations in adolescents with anxiety disorders. Hence, a brief introduction to common cognitive errors was considered of potential use.

We developed a video containing information on breathing exercises, information about cognitive errors and problem-solving skills. The video can be delivered using electronic media (for example on a television screen) in waiting rooms. The video starts with an explanation of breathing exercises, with an explanation of the fight and flight centre and how the centre is activated during stress, and explaining the breathing method in detail. The major part of the video revolves around a fictional character who uses steps in solving a problem to sort out a financial problem. These steps include defining the problems, making a list of problems, choosing the right solution and acting on it in small steps. Finally, the narrator explains common cognitive errors, each with an image and an example. This intervention is only $8 \mathrm{~min} 38 \mathrm{~s}$ long and can be shown while patients are in a waiting room in a psychiatric crisis clinic. It can be accessed at: (https://www.youtube.com/ watch? $=53 \mathrm{BA} 713 \mathrm{v} 6 \mathrm{ZA} \& \mathrm{t}=53 \mathrm{~s})$.

\section{Treatment as usual}

All study participants received treatment as usual (TAU), which mainly consisted of weekly or biweekly contacts with a caseworker who usually worked with the clients to monitor mental state, ensure medication adherence, help with practical issues such as housing, etc. Social workers and psychiatrist also supported clients.

\section{Method}

\section{Objectives}

The objectives of the study were to test the trial procedure and obtain outcome data to inform future definitive trials.

\section{Study design and setting}

This proof-of-concept study used a randomised, single-blind, controlled trial design (trial registration at Clinicaltrials.gov 
NCT02536924, REB Number: PSIY-477-15). It was conducted from January 2016 to January 2017. The local institutional review board at Queens University, Kingston, Canada, approved the trial protocol. After a full description of the study, all participants provided written informed consent before entering the study. The intervention group received RESOLVE and TAU, and the control group received TAU.

\section{Inclusions and exclusion criteria}

Individuals were included if they were $\geq 18$ years, were attending crisis service for the first time and had a diagnosis of a mood or anxiety disorder according to the DSM-IV. ${ }^{26}$ Individuals with substance dependence, active psychosis, organic brain syndrome or intellectual disability, as well as those with high levels of disturbed behaviour, were excluded.

\section{Participants}

Participants were recruited for the study during their visit to the crisis team walk-in services in Kingston, Ontario, Canada. Following their appointment with a crisis worker, participants were asked by their worker if they would like to participate in a research project. A member of the research team then contacted those considered suitable and invited them to participate in the study. Consenting participants were randomly allocated to intervention or control in a 1:1 ratio.

We enrolled 40 individuals, assigned randomly to the intervention group $(n=20)$ or the control group $(n=20)$. Three individuals from the intervention and four from the control group were not contactable for final assessment (see Fig. 1).

\section{Procedures}

Clinical staff identified 74 individuals who could potentially meet the inclusion criteria for the study. Research staff contacted this group, and 54 expressed interest in participating in the study. Out of 44 individuals considered suitable after the initial assessment, 40 individuals consented to and participated in the study (Fig. 1). Participants in the intervention arm were invited to watch the video in one of the assessment rooms adjacent to the waiting room. They were then given a copy of the video and handouts on problem-solving, relaxation and cognitive errors. Those in the control arm were offered only routine care.

Individuals who met inclusion criteria were randomly assigned to either the intervention $(n=20)$ or control $(n=20)$ only. Randomisation was performed using computer-generated numbers from a website (www.randomization.com). Block randomisation with randomly permuted block size was used to ensure similar numbers of participants were allocated to each arm of the trial. Individuals were assigned to either intervention or the control arm by the research team members who were independent of the assessment team.

\section{Outcome measures}

Outcome assessments were carried out by raters who were independent of those providing the intervention and masked to the intervention allocation and adherence. At the 6-week follow-up, participants had the option of completing the assessments in person or over the phone.

The following assessments were performed at baseline and 6 weeks; the Hospital Anxiety and Depression Scale (HADS), ${ }^{25}$ the Clinical Outcomes in Routine Evaluations (CORE) outcome measure, ${ }^{28}$ the World Health Organization Disability Assessment Schedule (WHO-DAS) $2.0^{27}$ and documentation of the number of contacts with the mental health services during the prior 4 weeks. The CORE was used to capture a broader range of symptoms and to see whether the change in psychopathology went above and beyond the anxiety and depressive symptoms. Finally, we decided to measure the change in disability to find out whether symptomatic change, if any, accompanied improvement in functioning or not. We also wanted to see whether this intervention will reduce the frequency of contacts with the service and therefore looked into this variable independently.

The $\mathrm{HADS}^{27}$ is a 14 item, self-assessment scale designed to measure anxiety and depression. It has high internal consistency, face validity and concurrent validity. Even numbered questions relate to depression and odd numbered questions relate to anxiety. Each question has four possible responses. Responses are scored on a scale from 0 to 3 . The maximum score is 21 for depression and 21 for anxiety. A score of 11 or higher indicates the probable presence of depression, whereas a score of 8 to 10 is suggestive of the presence of the respective state. In its current form the HADS is now divided into three ranges: normal $(0-7)$, borderline abnormal (8-10), 'cases' (11-21).

The brief WHO-DAS $2.0^{28}$ is a 12 -item self-report questionnaire that assesses disability and functioning in the prior month. The WHO-DAS 2.0 was developed to assess six different adult life tasks: (a) understanding and communication; (b) self-care; (c) mobility (getting around); (d) interpersonal relationships (getting along with others); (e) work and household roles (life activities); and (f) community and civic roles (participation).

The CORE outcome measure ${ }^{29}$ is a self-report questionnaire that asks 34 questions about how a person has been feeling over the past week. It uses a five-point scale ranging from 'not at all' to 'most or all of the time'. The 34 items of the measure cover four dimensions: subjective well-being, problems/symptoms, life functioning, risk/harm.

\section{The sample size}

This is a proof-of-concept study using an randomised control trial design. Therefore, no power calculation was conducted. The results of this proof-of-concept study will be used to calculate sample sizes for future larger randomised controlled trials. It has been suggested that a sample size of 12 in each arm should suffice for pilot studies. ${ }^{30}$ Allowing for up to $40 \%$ drop-out, we, therefore, recruited 40 participants for the study, including 20 participants per arm.

\section{Statistical analyses}

The analyses were carried out using SPSS v24. A descriptive analysis of the randomisation groups relative to its baseline characteristics was conducted where means and standard deviations were calculated for continuous variables and proportions for categorical variables. Because of the small sample sizes, group comparison at baseline was conducted using Fisher's exact test for categorical variables and the Mann-Whitney $U$-test for continuous variables. Our main analysis compared the outcomes at 6 weeks using the ANCOVA approach where the outcome at week 6 is specified as the dependent variable, and the treatment group along with baseline value of the outcome are specified as predictors. As this is a proofof-concept study, we have not adjusted $P$-values for multiple comparisons and used only data for completers in our main analysis. A sensitivity analysis that imputed outcomes using last-observation-carried-forward (LOCF) obtained almost identical results. In our case, we consider the LOCF approach conservative as most participants were observed to improve over time and LOCF necessarily forces the outcome to be stable over time. For practical interpretation, we also report raw Cohen's $d$ effect sizes. 


\section{Results}

Participants had a mean age of 30.3 years (range 20-52), with an almost equal gender ratio with $53 \%$ women $(n=21)$. They had a mean of 2.45 (range $0-10$ ) past admissions in a psychiatric facility. Most clients were ethnically White $(n=32,80 \%)$, were single $(n=$ $21,52.5 \%)$ and had college education $(n=26,65 \%)$. In terms of diagnoses, $30.0 \%(n=12)$ had depression, $10.0 \%(n=4)$ had generalised anxiety disorder, $12.5 \%(n=5)$ had post-traumatic stress disorder, $25 \%(n=10)$ had mixed anxiety and depression, $2.5 \%(n=1)$ had a personality disorder, $7.5 \%(n=3)$ had an anxiety disorder, $7.5 \%(n=3)$ had an adjustment disorder and 5\% $(n=2)$ had more than one diagnosis.

At baseline $40.0 \% \quad(n=16)$ were using antidepressants. Percentages for other baseline medications were 2.5\% $(n=1)$ anxiolytics, $2.5 \%(n=1)$ antipsychotics, $35.0 \%(n=14)$ mood stabilisers and $20.0 \%(n=8)$ were on multiple medication. Baseline demographics and clinical characteristics of the individuals in the intervention and control groups are shown in Table 1 . There were no statistical differences in any baseline values between the two groups.

Table 2 shows the results of the ANCOVA. Both the differences at the end of the intervention period and differences controlled for baseline are described. The participants in the treatment group showed significantly greater improvements on total HADS score $(P<0.001)$, anxiety subscale of HADS $(P<0.001)$, depression subscale of HADS $(P \leq 0.001)$, CORE $(P \leq 0.001)$ and disability $(P=$ 0.036 ) from baseline compared with the participants in the control group at the end of the study period. Participants in the intervention group also made fewer contacts with the mental health professionals during the past 4 weeks at the end of the study period (intervention, median 1.0 (interquartile range $(\mathrm{IQR})=3.0$ ), control mean 4.0 $(\mathrm{IQR}=2.0), P<0.001)$ as per the electronic health record system.

We performed a sensitivity analysis for each outcome with missing values at the end of the trial imputed using the LOCF method. Results are shown in Table 3. None of the conclusions mentioned previous changed.

\section{Discussion}

To the best of our knowledge, this is the first report of the development and pilot testing of an intervention using a waiting-room setting and based on an evidence-based therapy for common mental disorders. Those receiving the intervention showed improvement in depression, anxiety, general psychopathology and disability. These participants were also less likely to seek professional help compared with those in the control group. This has potential implications for healthcare costs. These initial results are encouraging and provide new opportunities for providing brief psychosocial interventions to people in a waiting room. Although we tested the intervention in a crisis clinic setting, it has the potential to be used in general psychiatric waiting rooms as well as in general medical waiting rooms or primary care settings.

There is significant prior evidence for the effectiveness of PST, mindfulness breathing and teaching cognitive errors for common mental disorders. This report confirms prior studies that PST can be effective for a variety of conditions, including psychiatric and physical health problems.

This work is in line with past reports of the success of singlesession psychological interventions. One session of cognitive behavioural therapy has been used to treat trauma ${ }^{12,13}$ anxiety for depression, ${ }^{31}$ for medication adherence for depression, ${ }^{32}$ for anxiety disorders, ${ }^{11,33,34}$ for pain catastrophizing ${ }^{35}$ and for addiction problems. ${ }^{36}$ Similarly, a single session of motivational
Table 1 Differences in demographic variables and psychopathology

between the intervention and the control groups at baseline

\begin{tabular}{|c|c|c|c|}
\hline & $\begin{array}{l}\text { Intervention } \\
(n=20)\end{array}$ & $\begin{array}{l}\text { Control (TAU) } \\
\quad(n=20)\end{array}$ & $P^{a}$ \\
\hline Age, mean (s.d.) & $28.8(10.7)$ & $31.2(9.4)$ & $<0.081$ \\
\hline $\begin{array}{l}\text { Past history of psychiatric } \\
\text { admissions, mean (s.d.) }\end{array}$ & $2.60(2.21)$ & $2.30(3.1)$ & $<0.461$ \\
\hline \multicolumn{4}{|l|}{ Gender, $n(\%)$} \\
\hline Men & $9(45)$ & $10(50)$ & \multirow[t]{2}{*}{$<1.000$} \\
\hline Women & $11(55)$ & $10(50)$ & \\
\hline \multicolumn{4}{|l|}{ Ethnicity, $n$ (\%) } \\
\hline White & $16(80)$ & $16(80)$ & \multirow[t]{2}{*}{$<1.000$} \\
\hline Black and minority ethnic & $4(20)$ & $4(20)$ & \\
\hline \multicolumn{4}{|l|}{ Marital status, $n(\%)$} \\
\hline Married or in a partnership & 7 (35) & $12(60)$ & \multirow[t]{2}{*}{$<0.205$} \\
\hline Single & $13(65)$ & $8(40)$ & \\
\hline \multicolumn{4}{|l|}{ Education, $n$ (\%) } \\
\hline High school & $1(5)$ & $4(20)$ & \multirow[t]{3}{*}{$<0.421$} \\
\hline College & $14(70)$ & $12(60)$ & \\
\hline Graduates or above & $5(25)$ & $4(20)$ & \\
\hline \multicolumn{4}{|l|}{ Past drug/alcohol use, $n$ (\%) } \\
\hline Recreational & $9(45)$ & $5(25)$ & \multirow[t]{4}{*}{$<0.076$} \\
\hline Misuse & $1(5)$ & $2(10)$ & \\
\hline Dependence & $1(5)$ & $7(35)$ & \\
\hline None & $9(45)$ & $6(30)$ & \\
\hline \multicolumn{4}{|l|}{ Employment, $n$ (\%) } \\
\hline Unemployed & $8(40)$ & $13(65)$ & \multirow[t]{2}{*}{$<0.205$} \\
\hline Employed & $12(60)$ & $7(35)$ & \\
\hline \multicolumn{4}{|l|}{ Diagnoses, $n$ (\%) } \\
\hline Depression & 7 (35) & $5(25)$ & \multirow[t]{8}{*}{$<0.445$} \\
\hline Generalised anxiety disorder & $2(10)$ & $2(10)$ & \\
\hline Post-traumatic stress disorder & $4(20)$ & $1(5)$ & \\
\hline Mixed anxiety and depression & $3(15)$ & $7(35)$ & \\
\hline Personality disorder & $0(0)$ & $1(5)$ & \\
\hline Anxiety disorder & $2(10)$ & $1(5)$ & \\
\hline Adjustment disorder & $2(10)$ & $1(5)$ & \\
\hline More than one diagnoses & $0(0)$ & $2(10)$ & \\
\hline \multicolumn{4}{|l|}{ Family history, ${ }^{b} n(\%)$} \\
\hline Yes & $14(70)$ & $12(60)$ & \multirow[t]{2}{*}{$<0.741$} \\
\hline No & $6(30)$ & $8(40)$ & \\
\hline \multicolumn{4}{|l|}{ Medication, $n$ (\%) } \\
\hline Antidepressants & $9(45)$ & $7(35)$ & \multirow[t]{5}{*}{$<0.644$} \\
\hline Anxiolytics & $0(0)$ & $1(5)$ & \\
\hline Antipsychotics & $0(0)$ & $1(5)$ & \\
\hline Mood stabilisers & $6(30)$ & $8(40)$ & \\
\hline Multiple medication & $5(25)$ & $3(15)$ & \\
\hline HADS Anxiety, mean (s.d.) & $11.25(1.74)$ & $10.75(3.04)$ & $<0.512$ \\
\hline HADS Depression, mean (s.d.) & $10.10(1.74)$ & $9.25(2.22)$ & $<0.289$ \\
\hline HADS Total, mean (s.d.) & $21.35(2.41)$ & $20(4.5)$ & $<0.414$ \\
\hline CORE, mean (s.d.) & 74.25 (19.81) & $69.45(21.60)$ & $<0.355$ \\
\hline WHO-DAS, mean (s.d.) & $23.05(6.40)$ & $21.45(5.28)$ & $<0.341$ \\
\hline \multicolumn{4}{|c|}{$\begin{array}{l}\text { TAU, treatment as usual; HADS, Hospital Anxiety and Depression Scale; CORE, Clinical } \\
\text { Outcomes in Routine Evaluations outcome measure; WHO-DAS, World Health } \\
\text { Organization Disability Assessment Schedule } 2.0 \text {. } \\
\text { a. P-values were calculated using non-parametric Mann-Whitney U-test for continuous } \\
\text { variables (age and clinical matrices) and Fisher's exact test for the rest of variables that } \\
\text { were categorical. } \\
\text { b. Family history of any psychiatric diagnosis. }\end{array}$} \\
\hline
\end{tabular}

interviewing has been shown to be effective for the treatment of addiction as well as in treatment adherence. ${ }^{14,15,36}$ Single-session interventions have also been tested and found to be effective using virtual reality programs for anxiety disorders. ${ }^{17,37}$

We aimed to develop a similar intervention using a video and the time spent in the waiting area. We found the intervention to be useful for the management of psychological distress. This might prove to be a cost-efficient way of delivering an intervention that is likely to be useful for a variety of conditions including psychiatric and physical health conditions. The participants for this trial were selected from a crisis team but the intervention could also be used in other settings such as waiting rooms for community mental health teams or primary care. 
Table 2 Differences between the treatment and control groups, both uncontrolled and controlled for baseline differences

\begin{tabular}{|c|c|c|c|c|c|c|c|}
\hline & \multicolumn{2}{|c|}{$\begin{array}{l}\text { Post-trial unadjusted group } \\
\text { means (s.d.) post trial }\end{array}$} & \multicolumn{5}{|c|}{ Mean differences adjusted for baseline outcome } \\
\hline & $\begin{array}{l}\text { Intervention } \\
\quad(n=17)\end{array}$ & $\begin{array}{c}\text { TAU } \\
(n=16)\end{array}$ & $\begin{array}{l}\text { Mean difference between } \\
\text { groups post trial }(95 \% \mathrm{Cl})\end{array}$ & Partial $\eta^{2}$ & (numDF, denDF) $F$ & Cohen's $d^{\mathrm{a}}$ & $P^{b}$ \\
\hline HADS Anxiety & $5.3(1.7)$ & $8.4(2.6)$ & $-3.6(-4.7$ to -2.5$)$ & 0.60 & (1.30) 45.2 & 1.65 & $<0.001$ \\
\hline HADS Depression & $3.9(2.2)$ & $7.0(2.1)$ & $-3.8(-5.0$ to -2.6$)$ & 0.57 & (1.30) 40.3 & 1.77 & $<0.001$ \\
\hline HADS Total & $9.2(3.7)$ & $15.4(4.2)$ & $-7.5(-9.7$ to -5.2$)$ & 0.51 & (1.30) 46.4 & 1.90 & $<0.001$ \\
\hline CORE & $31.1(20.7)$ & $57.3(18.9)$ & $-25.3(-39.8$ to -10.7$)$ & 0.29 & (1.30) 12.5 & 1.27 & 0.001 \\
\hline WHO-DAS 2.0 & $11.8(8.7)$ & $19.7(11.3)$ & $-7.9(-15.3$ to -0.5$)$ & 0.14 & (1.30) 4.8 & 0.80 & 0.036 \\
\hline \multicolumn{8}{|c|}{$\begin{array}{l}\text { TAU, treatment as usual; numDF, numerators for degrees of freedom; denDF, denominators for degrees of freedom; HADS, Hospital Anxiety and Depression Scale; CORE, Clinical Outcomes } \\
\text { in Routine Evaluations outcome measure; WHO-DAS, World Health Organization Disability Assessment Schedule } 2.0 \text {. } \\
\text { a. Cohen's } d \text { was calculated by dividing the adjusted difference at the end of the trial by the pooled SD at baseline. } \\
\text { b. P-values calculated using ANCOVA, controlling for baseline value of the outcome. }\end{array}$} \\
\hline
\end{tabular}

\begin{tabular}{|c|c|c|c|c|c|c|c|}
\hline & \multicolumn{2}{|c|}{$\begin{array}{l}\text { Post-trial unadjusted group } \\
\text { mean (s.d.) }\end{array}$} & \multicolumn{5}{|c|}{ Mean differences adjusted for baseline outcome } \\
\hline & Intervention $(n=20)$ & $\mathrm{TAU}(n=20)$ & $\begin{array}{c}\text { Mean difference } \\
\text { between groups at } \\
\text { post trial }(95 \% \mathrm{Cl})\end{array}$ & Partial $\eta^{2}$ & (numDF, denDF) $F$ & Cohen's $d^{\text {b }}$ & $P^{\mathrm{C}}$ \\
\hline HADS Anxiety & $6.1(2.6)$ & $8.9(2.6)$ & $-2.9(-4.5$ to -1.40$)$ & 0.29 & (1.37) 14.8 & 1.12 & $<0.001$ \\
\hline HADS Depression & $4.8(2.9)$ & $7.4(2.0)$ & $-3.1(-4.6$ to -1.6$)$ & 0.32 & (1.37) 17.9 & 1.24 & $<0.001$ \\
\hline HADS Total & $11.0(5.5)$ & $16.3(4.1)$ & $-6.0(-9.0$ to -2.0$)$ & 0.30 & (1.37) 16.5 & 1.24 & $<0.001$ \\
\hline CORE & $37.5(24.7)$ & $59.6(17.4)$ & $-21.6(-35.5$ to -7.7$)$ & 0.21 & (1.37) 9.9 & 1.01 & 0.003 \\
\hline WHO-DAS 2.0 & $13.5(8.9)$ & $19.9(10.0)$ & $-6.6(-12.8$ to -0.4$)$ & 0.11 & (1.37) 4.6 & 0.70 & 0.038 \\
\hline \multicolumn{8}{|c|}{$\begin{array}{l}\text { TAU, treatment as usual; numDF, numerators for degrees of freedom; denDF, denominators for degrees of freedom; HADS, Hospital Anxiety and Depression Scale; CORE, Clinical Outcomes } \\
\text { in Routine Evaluations outcome measure; WHO-DAS, World Health Organization Disability Assessment Schedule } 2.0 \text {. } \\
\text { a. Last-observation carried-forward was used to impute missing values for the seven patients who dropped out. Differences between the treatment and control groups, both uncontrolled } \\
\text { and controlled for baseline differences. Analyses were carried out using an ANCOVA. } \\
\text { b. Cohen's } d \text { was calculated by dividing the adjusted difference at end of trial by the pooled standard deviation at baseline. } \\
\text { c. P-values calculated using ANCOVA, controlling for baseline value of the outcome. }\end{array}$} \\
\hline
\end{tabular}

We believe that the waiting room could offer a suitable venue for providing psychosocial interventions. Patients spend a varying amount of time in waiting areas before healthcare professionals see them. This is the time when patients may be more receptive, and an intervention can not only help overcome anxiety in the waiting room but also can have a long-term beneficial effect. This may explain the significant effect of the intervention on depressive and anxiety symptoms

\section{Limitations}

Limitations include the small sample size and a short follow-up period. As such, more data is warranted to confirm these results. Participants in the intervention arm were invited individually to a small room adjacent to the waiting room to watch the video. In real life, not all those sitting in the waiting room might be receptive to the intervention. It is of note that the effect sizes were larger for psychopathology than disability. We also recognise that the large effect sizes might be because of the small sample size. Although this is a small study, future enhancements in the intervention could include behavioural activation to improve functional outcomes. Future studies need to include those with severe mental illness, and issues with substance misuse - the intervention in its current form was only tested with people with more common mental disorders. Similarly, the intervention was tested in a crisis clinic, although we believe it can be easily used in other medical and psychiatric settings.

Implications

Patients spend a significant amount of their time in psychiatric waiting rooms. This study confirms past reports of the efficacy of a single-session psychosocial intervention. However, the intervention in this group was delivered through a video instead of a therapist.
In this proof-of-concept study, we found that evidence-based interventions could be delivered through electronic media to a group of patients in the waiting room of a crisis team. We found that the intervention was acceptable and significantly improved anxiety and depressive symptoms. Further research is required to validate these findings with adequately powered trials with better methodology.

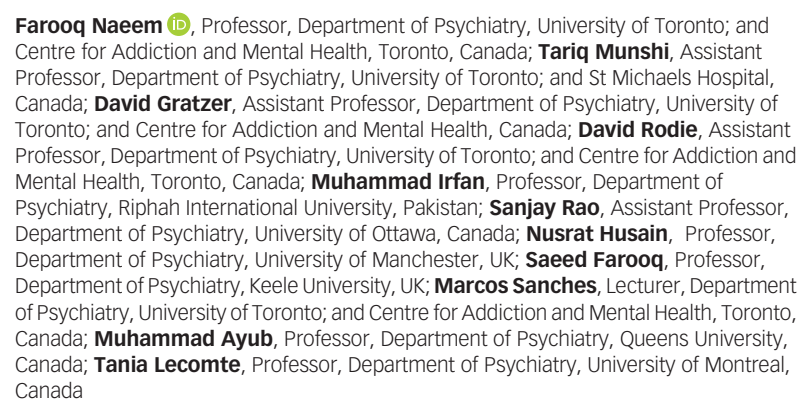

Canada

Correspondence: Faroog Naeem, Department of Psychiatry, University of Toronto, 33 Russell Street, Toronto, Canada. Email: faroognaeem@yahoo.com

First received 1 Sep 2018, final revision 28 Jun 2019, accepted 16 Jul 2019

\section{Funding}

The study was funded by a small internal grant by the Department of Psychiatry, Queens University, Kingston, Ontario.

\section{Acknowledgements}

We acknowledge all the participants. We also acknowledge Chris Trimmer, Richard Tyo, Hanna Taalman for collecting data. We are grateful to Tania Leverty and other members of the team for facilitating the study. 


\section{References}

1 Barlow GL. Auditing hospital queuing. Manag Audit J 2002; 17: 397-403.

2 Vitals.com. Wait time. Vitals, 2015 (http://vitalschoice.vitals.wpengine.com/ wait-time).

3 Sherwin HN, McKeown M, Evans MF, Bhattacharyya OK. The waiting room 'wait'. Can Fam Physician 2013; 59: 479-81.

4 Assathiany R, Kemeny J, Sznajder M, Hummel M, Van Egroo LD, Chevallier B, et al. The pediatrician's waiting room: a place for health education? [in French]. Arch Pediatr Organe Off Soc Francaise Pediatr 2005; 12: 10-5.

5 Eubelen C, Brendel F, Belche J-L, Freyens A, Vanbelle S, Giet D. Effect of an audiovisual message for tetanus booster vaccination broadcast in the waiting room. BMC Fam Pract 2011; 12: 104.

6 Koperski M. Health education using video recordings in a general practice waiting area: an evaluation. J R Coll Gen Pract 1989; 39: 328-30.

7 Oermann $\mathrm{MH}$, Webb SA, Ashare JA. Outcomes of videotape instruction in clinic waiting area. Orthop Nurs 2003; 22: 102-5.

8 Schwartz AL, Edelmann RJ. Health education: using a video in general practice. Br J Gen Pract 1990; 40: 477.

9 Wheeler JG, Fair M, Simpson PM, Rowlands LA, Aitken ME, Jacobs RF. Impact of a waiting room videotape message on parent attitudes toward pediatric antibiotic use. Pediatrics 2001; 108: 591-6.

10 Wicke $D$, Lorge R, Coppin R, Jones $K$. The effectiveness of waiting room noticeboards as a vehicle for health education. Fam Pract 1994; 11: 292-5.

11 Amir N, Bomyea J, Beard C. The effect of single-session interpretation modification on attention bias in socially anxious individuals. J Anxiety Disord 2010; 24: 178-82.

12 Başoğlu M, Şalcioğlu E, Livanou M. A randomized controlled study of single-session behavioural treatment of earthquake-related post-traumatic stress disorder using an earthquake simulator. Psychol Med 2007; 37: 203-13.

13 Bisson Jl. Single-session early psychological interventions following traumatic events. Clin Psychol Rev 2003; 23: 481-99.

14 Brand VS, Bray KK, MacNeill S, Catley D, Williams K. Impact of single-session motivational interviewing on clinical outcomes following periodontal maintenance therapy. Int J Dent Hyg 2013; 11: 134-41.

15 Diskin KM, Hodgins DC. A randomized controlled trial of a single session motivational intervention for concerned gamblers. Behav Res Ther 2009; 47: 382-8.

16 Fu X, Du Y, Au S, Lau JYF. Reducing negative interpretations in adolescents with anxiety disorders: a preliminary study investigating the effects of a single session of cognitive bias modification training. Dev Cogn Neurosci 2013; 4: 29-37.

17 Miloff $A$, Lindner $P$, Hamilton W, Reuterskiöld L, Andersson G, Carlbring $P$. Single-session gamified virtual reality exposure therapy for spider phobia vs. traditional exposure therapy: study protocol for a randomized controlled non-inferiority trial. Trials 2016; 17: 60 .

18 Hoffman YSG, Pitcho-Prelorentzos S, Ring L, Ben-Ezra M. 'Spidey can': preliminary evidence showing arachnophobia symptom reduction due to superhero movie exposure. Front Psychiatry 2019; Jun 7 (Epub ahead of print).

19 Naeem $F$, Munshi T, Xiang S, Yang M, Shokraneh F, Syed $Y$, et al. A survey of eMedia-delivered interventions for schizophrenia used in randomized controlled trials. Neuropsychiatr Dis Treat 2017; 13: 233-43.
20 Bell AC, D'Zurilla TJ. Problem-solving therapy for depression: a meta-analysis. Clin Psychol Rev 2009; 29: 348-53.

21 Xia J, Li C. Problem solving skills for schizophrenia. Cochrane Database Syst Rev 2007; (2): CD006365.

22 Bilsker D, Forster P. Problem-solving intervention for suicidal crises in the psychiatric emergency service. Crisis 2003; 24: 134-6.

23 Khoury B, Lecomte T, Gaudiano BA, Paquin K. Mindfulness interventions for psychosis: a meta-analysis. Schizophr Res 2013; 150: 176-84.

24 Ma X, Yue Z-Q, Gong Z-Q, Zhang H, Duan N-Y, Shi Y-T, et al. The effect of diaphragmatic breathing on attention, negative affect and stress in healthy adults. Front Psychol 2017; 8: 874.

25 Beck AT, Rush AJ, Shaw BF, Emery G. Cognitive Therapy of Depression (1st edn): 425. The Guilford Press, 1979.

26 American Psychiatric Association. Diagnostic and Statistical Manual of Mental Disorders (DSM-IV-TR) 4th edn, text revision. American Psychiatric Association, 2000

27 Zigmond AS, Snaith RP. The hospital anxiety and depression scale. Acta Psychiatr Scand 1983; 67: 361-70.

28 Üstün TB, Chatterji S, Kostanjsek N, Rehm J, Kennedy C, Epping-Jordan J, et al. Developing the world health organization disability assessment schedule 2.0. Bull World Health Organ 2010; 88: 815-23.

29 Evans C, Mellor-Clark J, Margison F, Barkham M, Audin K, Connell J, et al. CORE: clinical outcomes in routine evaluation. J Ment Health 2000; 9: 247-55.

30 Julious SA. Sample size of 12 per group rule of thumb for a pilot study. Pharm Stat 2005; 4: 287-91.

31 Kunik ME, Braun U, Stanley MA, Wristers K, Molinari V, Stoebner D, et al. One session cognitive behavioural therapy for elderly patients with chronic obstructive pulmonary disease. Psychol Med 2001; 31: 717-23.

32 Safren SA, O'Cleirigh C, Tan JY, Raminani SR, Reilly LC, Otto MW, et al. A randomized controlled trial of cognitive behavioral therapy for adherence and depression (CBT-AD) in HIV-infected individuals. Health Psychol 2009; 28: $1-10$.

33 De Jongh A, Muris P, Horst GT, Van Zuuren F, Schoenmakers N, Makkes P. Onesession cognitive treatment of dental phobia: preparing dental phobics for treatment by restructuring negative cognitions. Behav Res Ther 1995; 33: 947-54.

34 Öst L-G. One-session treatment for specific phobias. Behav Res Ther 1989; 27: $1-7$.

35 Darnall BD, Sturgeon JA, Kao M-C, Hah JM, Mackey SC. From catastrophizing to recovery: a pilot study of a single-session treatment for pain catastrophizing. J Pain Res 2014; 7: 219-26.

36 Copeland J, Swift W, Roffman R, Stephens R. A randomized controlled trial of brief cognitive-behavioral interventions for cannabis use disorder. J Subst Abuse Treat 2001; 21: 55-64.

37 Berman AH, Forsberg L, Durbeej N, Källmén H, Hermansson U. Single-session motivational interviewing for drug detoxification inpatients: effects on selfefficacy, stages of change and substance use. Subst Use Misuse 2010; 45: 384-402.

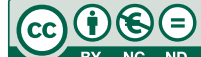

